\title{
Polar and Orbit Plot Analysis for Unbalance Identification in A Rotating System
}

\author{
Arka Sen ${ }^{1 *}$, Manik Chandra Majumder ${ }^{2}$, Sumit Mukhopadhyay ${ }^{2}$, \\ Robin Kumar Biswas ${ }^{3}$, \\ ${ }^{1} *{ }^{2}$ Department Of Mechanical Engineering, National Institute Of Technology Durgapur, West Bengal, India. \\ ${ }^{3}$ Condition Monitoring And Structural Analysis Department, Central Mechanical Engineering Research \\ Institute, Durgapur, India.
}

\begin{abstract}
This paper presents a detailed analysis of polar and orbit plots considering a non-faulty shaft and one with an unbalanced mass attached to it. Polar Plot analysis was done in order to determine the amplitude of vibration and the phase difference between faulty and non-faulty case. Also the orbit plot analysis provides a clear image of the two different cases, in which the area under the ellipse can be considered as the measure of the unbalance.
\end{abstract}

\section{Introduction}

Unbalance is defined as an unequal distribution of mass causing the mass axis to differ from the bearing axis. During rotation, the unequal mass along with the radial acceleration due to rotation create a centrifugal force. This results in force on the bearings and/or vibration of the bearings. Balancing is a procedure in which the mass distribution of a rotor is assessed and if necessary, adjusted via addition or subtraction of weight to ensure that the vibration of the journals and/or forces on the bearings are within specified limits. Vibration is a mechanical movement where an object oscillates about an equilibrium point. It commonly produces unwanted sound and wastes energy. Vibration in rotating equipment can greatly reduce the life of the equipment and the bearings. Before studying the basic principles of balancing, one must keep in mind that there are many causes of vibration other than unbalance. In some cases, balancing may result in only a partial or temporary reduction in vibration, while in other cases balancing is the only effective course of action.

Mechanical unbalance, which produces a force at 1 X RPM, has been found to be one of the most common causes of machinery vibration, present to some degree on nearly all rotating machines. Polar Plot and Orbit Plot analysis are methods to determine the unbalance in the machine. By the nature of the plots, one can easily determine the type of fault in the machine and can take remedial action immediately in order to prevent any catastrophic effects on the machine.

\subsection{Experimental setup and procedure of data collection:}

\section{Materials And Methodology}

Considering the main cause of rotating machine vibration as unbalance, a test rig for experimental validation of the model based identification technique was built. Schematic representation of the experimental set has been shown in the figures below.
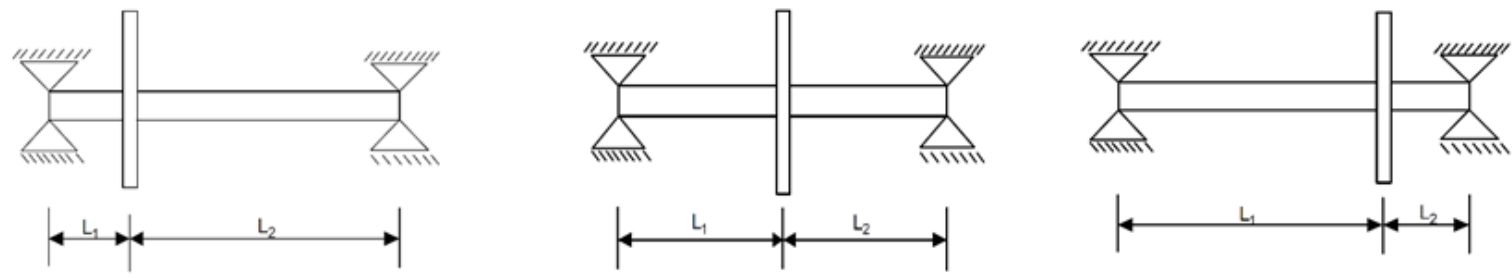

Figure 5: Schematic representation of the experimental set up (no fault case)
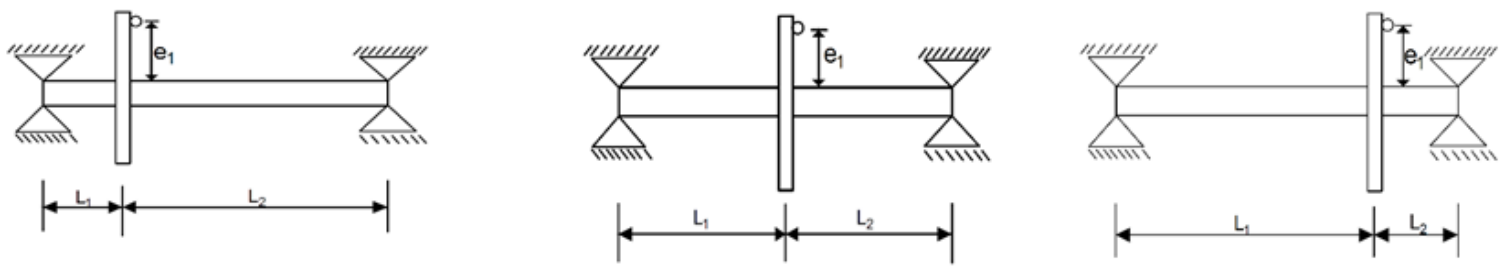

Figure 6: Schematic representation of the experimental set up (with unbalance fault) 
The experimental verification for the Unbalance Identification of mass on a shaft with single plane and two eccentricity location has been performed on a Machine Fault Simulator available at Central mechanical Engineering Research Institute (CSIR-CMERI) in Durgapur.

A rigid shaft considered to be massless is mounted between two roller bearings. The distance between the two bearings is $\mathrm{L}_{1}+\mathrm{L}_{2}$, which is $60 \mathrm{~cm}$. This shaft is connected to a Variable Frequency Drive (VFD) motor by a flexible coupling. To measure the vibration in X-Direction and Y-Direction at the two bearings, four accelerometers are connected; two in each bearing. The weight of the disc is 653 gram $\left(\mathrm{M}_{1}\right)$. The position of the disc is varied in three different locations: (i) $15 \mathrm{~cm}$ from left bearing (ii) $30 \mathrm{~cm}$ from left bearing which is the mid position on the shaft (iii) $45 \mathrm{~cm}$ from left bearing. Initially no unbalance mass was attached in order to get the no fault readings. Three unbalance masses of 8 gram $\left(\mathrm{m}_{1}\right), 12$ gram $\left(\mathrm{m}_{2}\right)$ and 16 gram $\left(\mathrm{m}_{3}\right)$ are attached subsequently to the disc at eccentricities $6.85 \mathrm{~cm}\left(\mathrm{e}_{1}\right)$ and $6.85 \mathrm{~cm}\left(\mathrm{e}_{2}\right)$ separately one by one. Then the shaft is rotated at rpm 300, 600, 900, 1200 and 1500; and simultaneously the vibration readings (RMS values) and their phase values in $\mathrm{x}$ and $\mathrm{y}$-direction at the two bearings were noted down. Artificial Neural Networking Techniques with varying training algorithmshave been used to determine the unbalance plane of vibration.

Mass Unbalance: The unbalance masses used are 8 gram, 12 gram and 16 gram which are shown in the figures below.

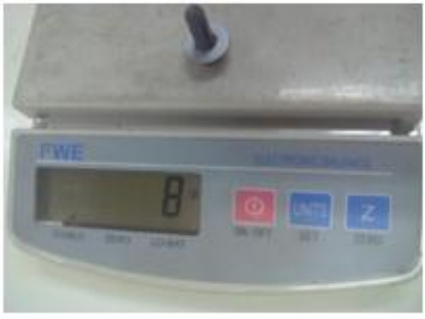

Fig 7

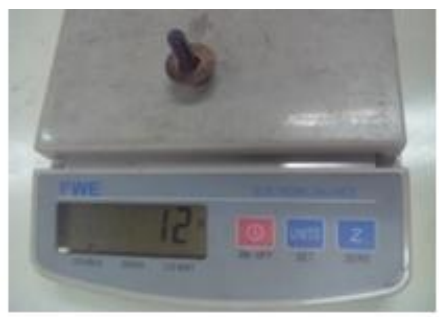

Fig 8

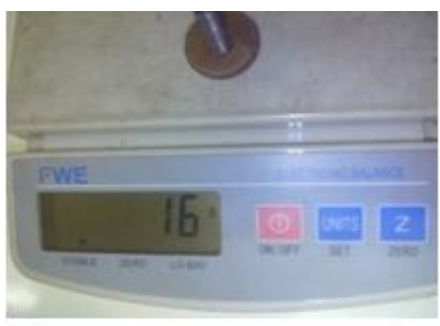

Fig 9

Fig 7: Unbalance mass of 8gram attached to the rotating system; Fig 8: Unbalance mass of 10gram attached to the rotating system; Fig 9: Unbalance mass of 8 gram attached to the rotating system

\section{Eccentricity:}

All rotors have some eccentricity. Eccentricity is present when geometrical centre of the rotor and the mass centre do not coincide along their length. In the present case, the disc is considered absolutely balanced where the Geometrical Centre of the disc coincides with the Centre of the Gravity of the disc. In the current research work eccentricity is the distance between the Geometrical Centre (Centre of rotation) and the points where the unbalance mass is to be attached. There are two locations at a distance of $6.85 \mathrm{~cm}$ from the centre of rotation $\left(\mathrm{e}_{1}\right)$ and at a distance of $4.85 \mathrm{~cm}$ from the centre of rotation of the disc $\left(\mathrm{e}_{2}\right)$.

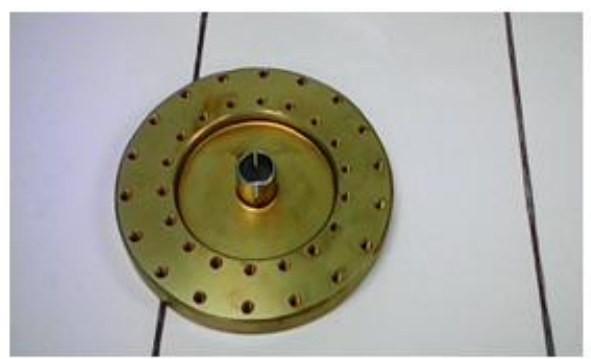

Fig 10: The rotating disc

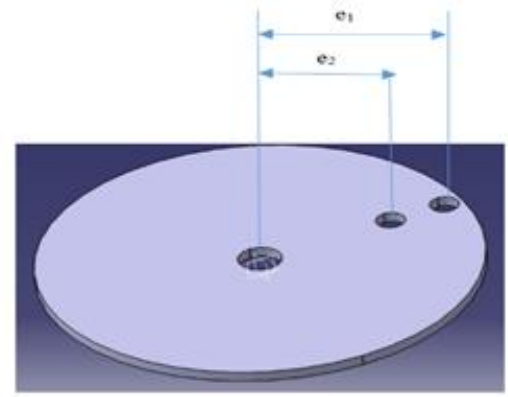

Fig 11: Schematic representation of rotating disc

\section{Plane of Unbalance:}

The Figure 13, Figure 14 and Figure 15 shows the different position of the rotating disc placed on the shaft (left, centre and right respectively). 


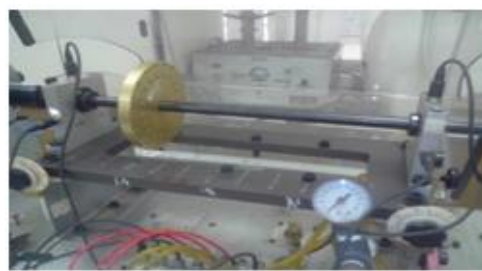

Fig 13

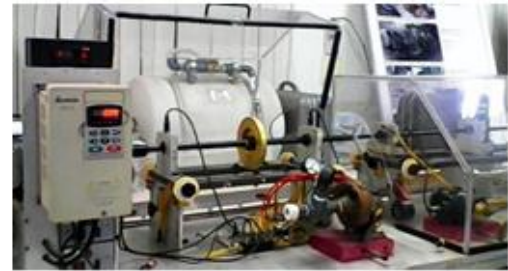

Fig 14

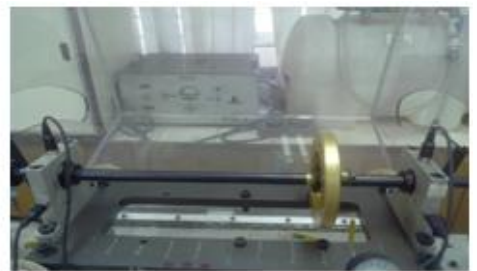

Fig 15

The design of experiment (Table 2) is based on the maximum possible combination of Unbalance mass $(\mathrm{m})$, Eccentricity (e), Plane of unbalance (p) and the RPM (r) values. The table (Table 1) below provides the maximum and minimum values the independent parameters/input to the system.

Table 1: Independent/Input parameters and their levels

\begin{tabular}{|c|c|c|c|}
\hline Serial No & Independent /Input Parameters & Minimum Level & Maximum Level \\
\hline 1 & Unbalance Mass (M) & $8 \mathrm{gram}$ & $16 \mathrm{gram}$ \\
\hline 2 & Eccentricity (E) & $4.85 \mathrm{~cm}$ & $6.85 \mathrm{~cm}$ \\
\hline 3 & Plane Of Unbalance (P) & $\begin{array}{c}15 \mathrm{~cm} \text { (Measured From } \\
\text { Left Bearing) }\end{array}$ & $\begin{array}{c}45 \mathrm{~cm} \text { (Measured } \\
\text { From Left Bearing) }\end{array}$ \\
\hline 4 & Revolutions Per Minute (Rpm) (R) & 300 & 1500 \\
\hline
\end{tabular}

Table 2: Design of Experiment

\begin{tabular}{|c|c|c|c|c|}
\hline Set & $\begin{array}{c}\text { Experimental } \\
\text { Codes }\end{array}$ & Unbalance Mass & Eccentricity & $\begin{array}{c}\text { Location Of Unbalance } \\
\text { Plane (In Cm) }\end{array}$ \\
\hline & & (In Gram) & $($ In Cm) & (From Left Bearing ) \\
\hline 1 & Nfl & 0 & 0 & 30 \\
\hline 2 & Nfc & 0 & 0 & 45 \\
\hline 3 & Nfr & 0 & 4.85 & 15 \\
\hline 4 & L8e2 & 8 & 4.85 & 30 \\
\hline 5 & C8e2 & 8 & 4.85 & 45 \\
\hline 6 & R8e2 & 8 & 6.85 & 15 \\
\hline 7 & L8e1 & 8 & 6.85 & 30 \\
\hline 9 & C8e1 & 8 & 6.85 & 45 \\
\hline 10 & R8e1 & 8 & 4.85 & 30 \\
\hline 11 & L12e2 & 12 & 4.85 & 45 \\
\hline 12 & C12e2 & 12 & 4.85 & 15 \\
\hline 13 & R12e2 & 12 & 6.85 & 30 \\
\hline 14 & L12e1 & 12 & 6.85 & 45 \\
\hline 15 & C12e1 & 12 & 6.85 & 15 \\
\hline 16 & R12e1 & 12 & 4.85 & 30 \\
\hline 17 & L16e2 & 16 & 4.85 & 45 \\
\hline 18 & C16e2 & 16 & 6.85 & 15 \\
\hline 19 & R16e2 & 16 & 6.85 & 30 \\
\hline 20 & L16e1 & 16 & 6.85 & 45 \\
\hline 21 & C16e1 & 16 & & \\
\hline
\end{tabular}

The design of experiment as mentioned in the table 2 has been performed for 300, 600, 900, 1200 and 1500 RPM`s. In total there are 105 set of experiments.

\subsection{Indicators Used In Experiment :}

NFC: No fault disc at Centre,NFL: No fault disc at Left position,NFR: No fault disc at Right position The number succeeding NFC, NFL, NFR represents the rpm at which the disc is rotating without any unbalance mass attached. With unbalance mass attached, the experimental code is written in the following format as mentioned below one after the other.

Position of Rotating Disc $(\mathrm{p}) \longrightarrow$ Unbalance Mass $(\mathrm{m}) \longrightarrow$ Eccentricity $(\mathrm{e}) \longrightarrow \quad$ Revolutions Per Minute (RPM) (r)

For example:C16e11500 represents the rotating disc placed at centre of the shaft with mass unbalance of 16 gram placed at an eccentricity $e_{1}$ rotated at 1500 RPM. It is similar for rest of the experimental cases.

\section{Results And Discussion:}

Polar Plot Analysis

A polar plot displays data in polar coordinates, which enables you to see phase changes in the range of zero to 360 degrees. The polar plot zero degree point always is located at the angular position of a transducer. You can 
compare data from orthogonally-mounted proximity probe pairs with a polar plot. Fig16-23 depicts the polar plots for a no fault case and a faulty case with 16 gram unbalance mass placed on the disc at eccentricity $\mathrm{e}_{1}$ located at the centre of the shaft.



Fig 16: Polar Plot for NFC1500 (Ch-2)

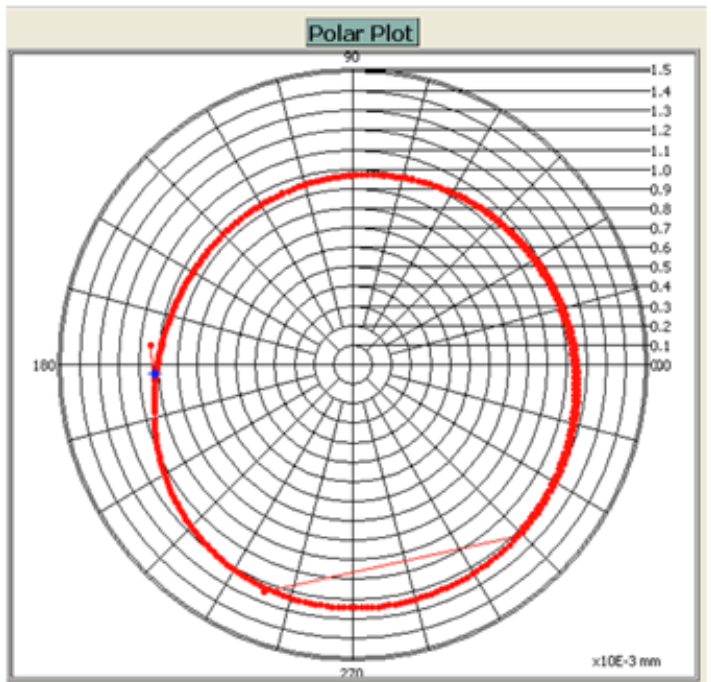

Fig 18: Polar Plot for NFC1500 (Ch-3)

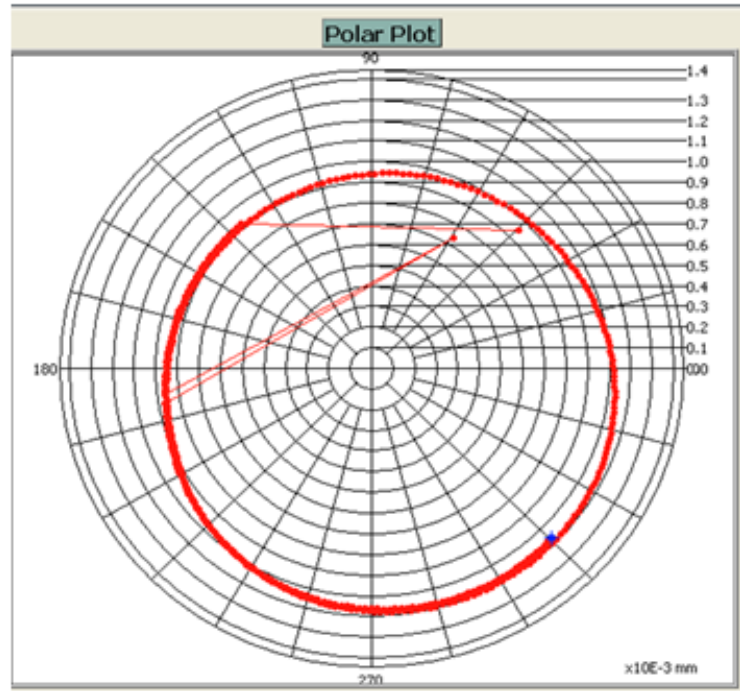

Fig 20: Polar Plot for NFC1500 (Ch-7)

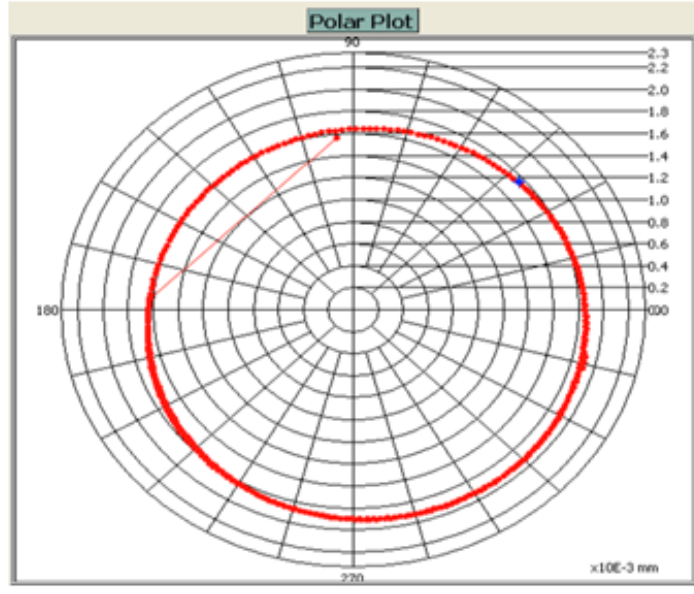

Fig 17: Polar Plot for C16E11500 (Ch-2)

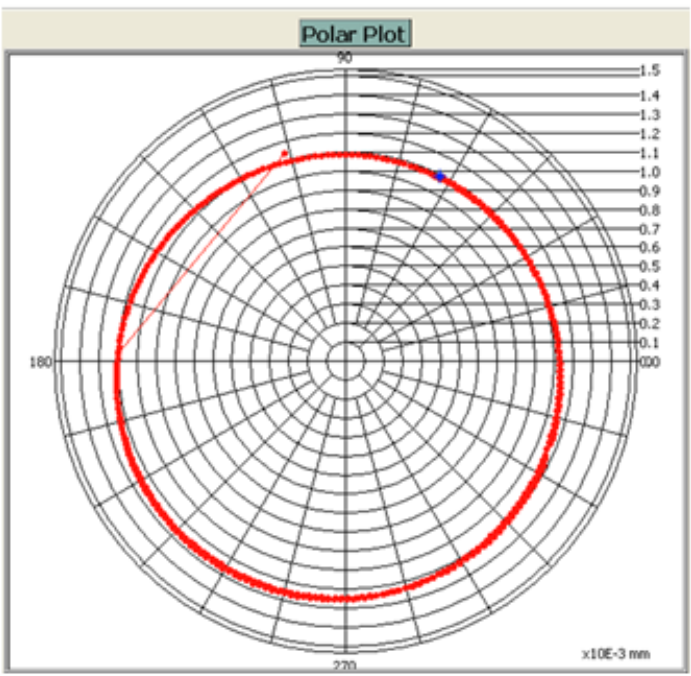

Fig 19: Polar Plot for C16E11500 (Ch-3)

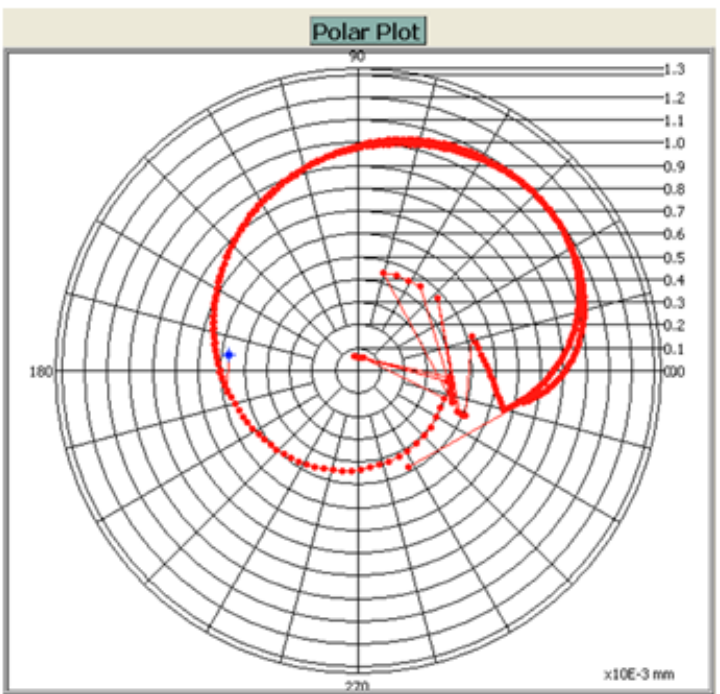

Fig 21: Polar Plot for C16E11500 (Ch-7) 


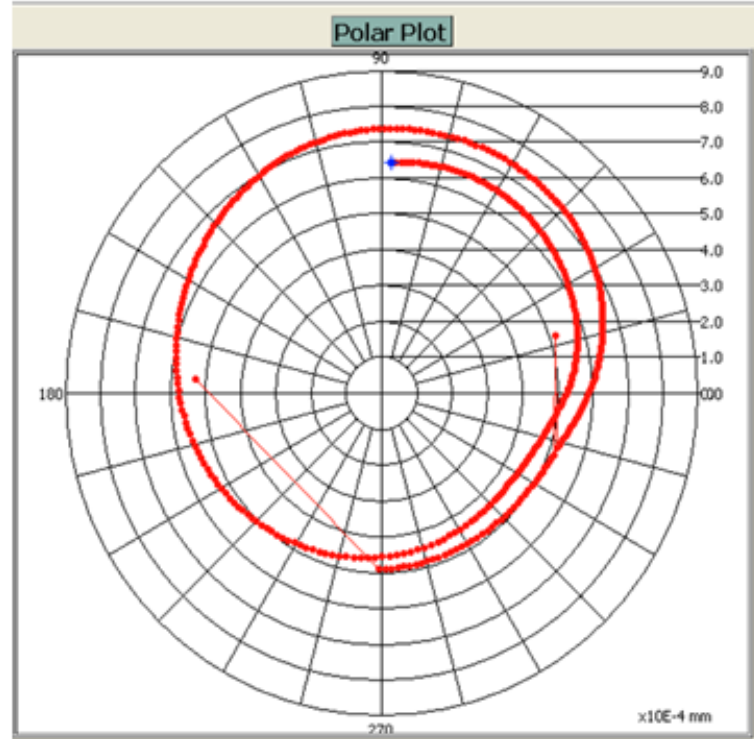

Fig 22: Polar Plot for NFC1500 (Ch-8)



Fig 23: Polar Plot for C16E11500 (Ch-8)

Table 3: Amplitude and Phase values from Polar Plot Analysis

\begin{tabular}{|l|l|l|l|l|l|l|l|l|}
\hline & \multicolumn{3}{|l|}{$\begin{array}{l}\text { Left Bearing X-Axis } \\
\text { (Channel-2) }\end{array}$} & \multicolumn{2}{l}{$\begin{array}{l}\text { Right Bearing X-Axis } \\
\text { (Channel-3) }\end{array}$} & \multicolumn{2}{l}{$\begin{array}{l}\text { Left Bearing Y-Axis } \\
\text { (Channel-7) }\end{array}$} & \multicolumn{2}{l|}{$\begin{array}{l}\text { Right Bearing Y-Axis } \\
\text { (Channel-8) }\end{array}$} \\
\hline & Amplitude & Phase & Amplitude & Phase & Amplitude & Phase & Amplitude & Phase \\
\hline $\begin{array}{l}\text { No Fault } \\
\text { Case }\end{array}$ & $1.54 \mathrm{E}-3$ & 166.79 & $1.02 \mathrm{E}-3$ & 182.66 & $1.18 \mathrm{E}-3$ & 315.49 & $6.49 \mathrm{E}-4$ & 87.70 \\
\hline C16E11500 & $1.77 \mathrm{E}-3$ & 41.27 & $1.10 \mathrm{E}-3$ & 63.13 & $2.73 \mathrm{E}-3$ & 172.94 & $1.18 \mathrm{E}-3$ & 337.11 \\
\hline
\end{tabular}

From the polar plot analysis, it is evident that there exists a phase difference of 90degrees for unbalance fault in each direction of the bearings. Proximity probes like Channel-2, Channel-7, Channel3 and Channel 8 have been set up to measure the vibrations in Left Bearing X, Left Bearing Y, Right Bearing X and Right Bearing Y direction respectively. The phase difference for Channel-2 and Channel -3 are 125.52 and 119.53 degree respectively which can be considered to be very close to each other. There is a little more difference in values for Channel-7 and Channel- 8 due to experimental error which is within the limits of acceptance.

\section{Orbit Plot Analysis}

The ellipticity of the filtered orbit, as well as the amplitude and the inclination angle of the major axis of the orbit, are parameters whose analysis can provide important diagnostic information

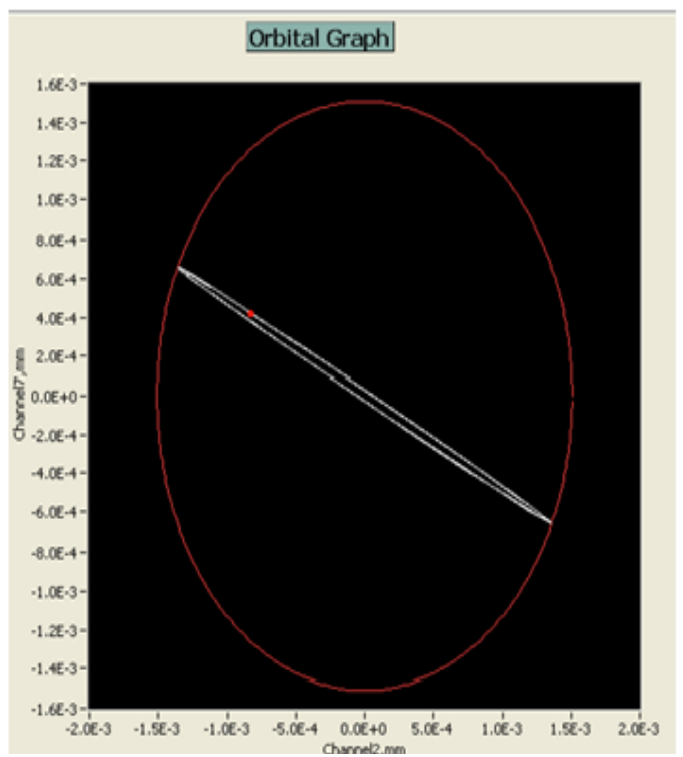

Fig 24: Orbit Plot for Ch-2 \& 7 (No fault case)

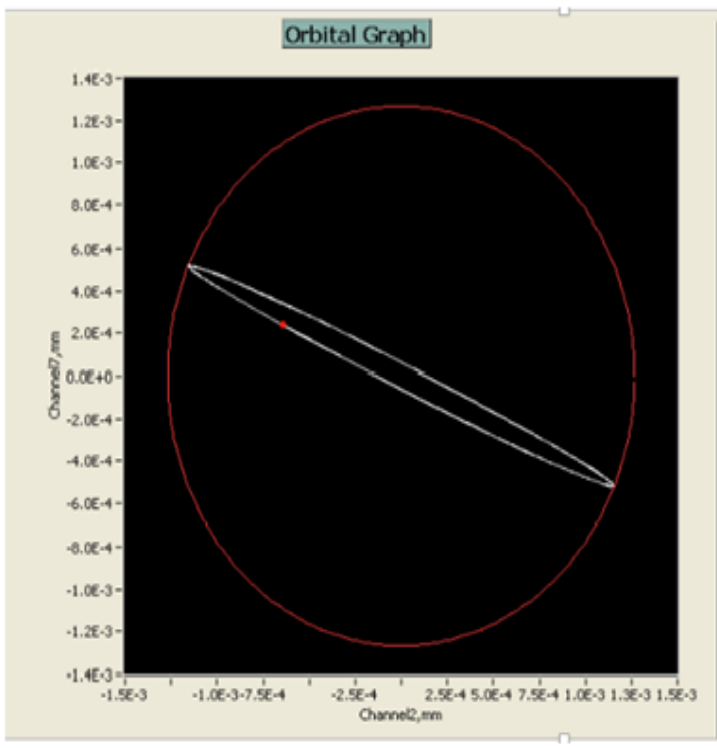

Fig 25: Orbit Plot for Ch-2 \&7 (C16E11500) 


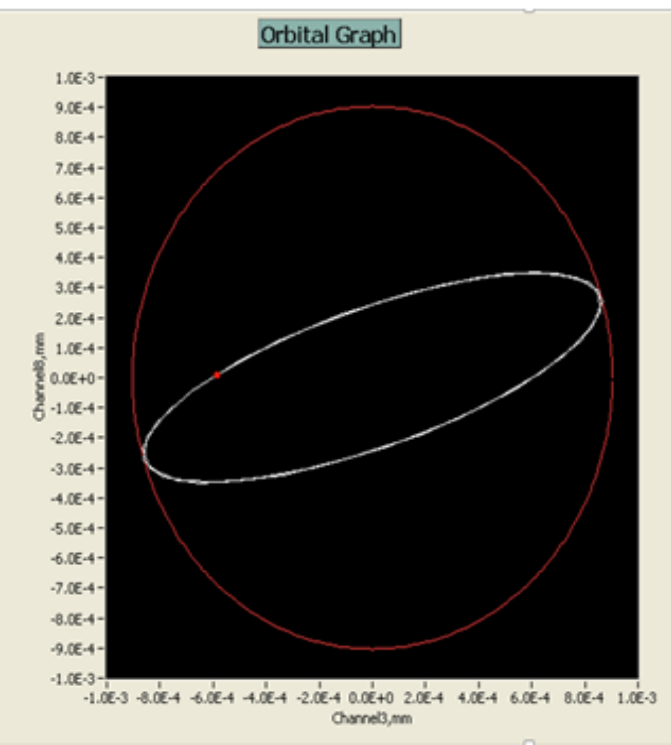

Fig 26: Orbit Plot for Ch-3 \& 8 (No fault case)

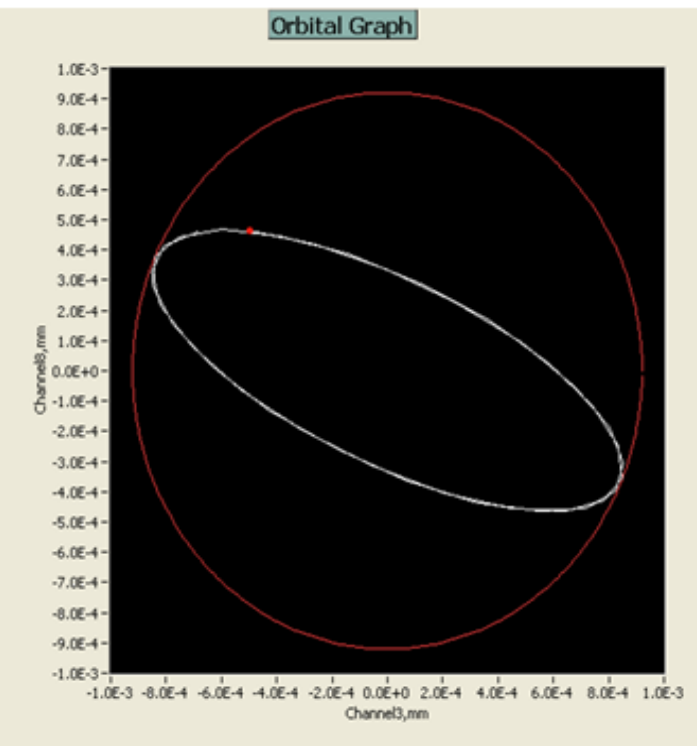

Fig 27: Orbit Plot for Ch-3 \&8 (C16E11500)

Table 4: Overall values from Orbit Plot Analysis

\begin{tabular}{|c|c|c|c|c|}
\hline & $\begin{array}{l}\text { Left Bearing X-Axis } \\
\text { (Channel-2) } \\
\text { (Overall values) }\end{array}$ & $\begin{array}{l}\text { Left Bearing Y-Axis } \\
\text { (Channel-7) } \\
\text { (Overall Values) }\end{array}$ & $\begin{array}{l}\text { Right Bearing X- } \\
\text { Axis (Channel-3) } \\
\text { (Overall Values) }\end{array}$ & $\begin{array}{l}\text { Right Bearing Y- } \\
\text { Axis (Channel-8) } \\
\text { (Overall Values) }\end{array}$ \\
\hline No Fault Case & .00231 & .00104 & .00171 & .0008 \\
\hline C16E11500 & .00271 & .00131 & .00173 & .000932 \\
\hline
\end{tabular}

From the orbit plot analysis, it is evident that the area of the ellipse for unbalance fault case ismore than that of the no fault case. From Table 4 , it can be seen that the overall values in case of the unbalance fault case is more as compared to its no fault case. From the shape of the curve in the orbit plot, the type of fault can be determined, which in this case is unbalance fault as because the plot is elliptical. Different shapes of plot indicates various faults in the rotating system.

\section{Conclusion}

A comparative polar and orbit plot analysis has been done for no fault and faulty case. Similarly, polar and orbit plots can be drawn for different other faults like misalignment, bearing faults etc with more than one disc mounted on the shaft and more than one unbalance mass attached to the system.

\section{Acknowledgement}

The author is thankful to the Department of Mechanical Engineering, National Institute of Technology Durgapur and Condition Monitoring and Structural Analysis Department of Central Mechanical Engineering Research Institute located at Durgapur to help me to carry out this research work on "Polar and Orbit Plot analysis for Unbalance Identification in a Rotating System".

\section{Reference}

[1]. R. J. Kuo, Intelligent Diagnosis for Turbine Blade Faults Using Artificial Neural Networks and Fuzzy Logic, Engineering Application Artificial Intelligence. Vol. 8, No. 1, pp. 25-34, 1995.

[2]. Yuan-Pin Shih and An-Chen Lee, Identification of the unbalance distribution in flexible rotors,Int. J. Mech. Sci. Vol. 39, No. 7, pp. $841857,1997$.

[3]. K N Gupta, Vibration - A tool for machine diagnostics and condition monitoring, Sadhana, Vol. 22, Part 3, June 1997, pp. 393-410.

[4]. Jae Hong Suh, Soundar R. T. Kumara (I), Shreesh P. Mysore, Machinery Fault Diagnosis and Prognosis: Application of Advanced Signal Processing Techniques, Annals of the CIRP Vol. 48/7/7999 317.

[5]. RoyaJavadpour, Gerald M. Knapp, A fuzzy neural network approach to machine condition monitoring, Computers \& Industrial Engineering 45 (2003) 323-330.

[6]. Jason R. Blough, A survey of DSP methods for rotating machinery analysis what is needed, what is available, Journal of Sound and Vibration 262 (2003) 707-720.

[7]. Peter W. Tse, Wen-Xian Yang, H.Y. Tam, Machine fault diagnosis through an effective exact wavelet analysis, Journal of Sound and Vibration 277 (2004) 1005-1024.

[8]. D.F. Shi, F. Tsung, P.J. Unsworth,Adaptive time-frequency decomposition for transient vibration monitoring of rotating machinery, Mechanical Systems and Signal Processing 18 (2004) 127-141.

[9]. Z.K. Peng, F.L. Chu, Application of the wavelet transform in machine condition monitoring and fault diagnostics: a review with bibliography, Mechanical Systems and Signal Processing 18 (2004) 199-221. 
[10]. YazhaoQiu, Singiresu S. Rao ,A fuzzy approach for the analysis of unbalanced nonlinear rotor systems , Journal of Sound and Vibration 284 (2005) 299-323

[11]. Bo-Suk Yang, Dong-Soo Lib, Andy Chit Chiow Tan, VIBEX: an expert system for vibration fault diagnosis of rotating machinery using decision tree and decision table, Expert Systems with Applications 28 (2005) 735-742

[12]. B Liu, Selection of wavelet packet basis for rotating machinery fault diagnosis, Journal of Sound and Vibration 284 (2005) 567582 .

[13]. Michael Feldman, Time-varying vibration decomposition and analysis based on the Hilbert transform, Journal of Sound and Vibration 295 (2006) 518-530

[14]. Jiangping Wang, Hongtao Hu, Vibration-based fault diagnosis of pump using fuzzy technique, Measurement 39 (2006) $176-185$.

[15]. Andrew K.S. Jardine, Daming Lin, DraganBanjevic, A review on machinery diagnostics and prognostics implementing conditionbased maintenance, Mechanical Systems and Signal Processing 20 (2006) 1483-1510.

[16]. Sheng-Fa Yuan, Fu-Lei Chu, Support vector machines-based fault diagnosis for turbo-pump rotor, Mechanical Systems and Signal Processing 20 (2006) 939-952.

[17]. Qiang Miao, ViliamMakis, Condition monitoring and classification of rotating machinery using wavelets and hidden Markov models, Mechanical Systems and Signal Processing 21 (2007) 840-855.

[18]. Javier Sanz, Ricardo Perer, Consuelo Huert, Fault diagnosis of rotating machinery based on auto-associative neural networks and wavelet transforms, Journal of Sound and Vibration 302 (2007) 981-999.

[19]. Qiao Hu, Z Zhengji He, Zhousuo Zhang, YanyangZi, Fault diagnosis of rotating machinery based on improved wavelet package transform and SVMs ensemble, Mechanical Systems and Signal Processing 21 (2007) 688-705.

[20]. Yaguo Lei, Zhengji He, YanyangZi, Qiao Hu , Fault diagnosis of rotating machinery based on multiple ANFIS combination with Gas, Mechanical Systems and Signal Processing 21 (2007) 2280-2294.

[21]. AiwinaHeng, Sheng Zhang, Andy C.C.Tan, Joseph Mathew, Rotating machinery prognostics: State of the art, challenges and opportunities, Mechanical Systems and Signal Processing23 (2009)724-739.

[22]. Ying Peng, Ming Dong, Ming JianZuo, Current status of machine prognostics in condition-based maintenance: a review, International Journal of Advanced Manufacturing Technology (2010) 50:297-313.

[23]. Chenxing Sheng, Zhixiong Li, Li Qin, ZhiweiGuo, Yuelei Zhang, Recent Progress on Mechanical Condition Monitoring and Fault diagnosis, Procedia Engineering 15 (2011) 142 - 146

[24]. Huo-Ching Sun, Yann-Chang Huang, Support Vector Machine for Vibration Fault Classification of Steam Turbine-Generator Sets, Procedia Engineering 24 (2011) $38-42$.

[25]. F. Al-Badour , M.Sunar , L.Cheded, Vibration analysis of rotating machinery using time-frequency analysis and wavelet techniques, Mechanical SystemsandSignalProcessing25(2011)2083-2101.

[26]. Lei You, Jun Hu, Fang Fang, LintaoDuan, Fault diagnosis system of rotating machinery vibration signal, Procedia Engineering 15 (2011) $671-675$.

[27]. XinXiong, Shixi Yang, ChunbiaoGan, A new procedure for extracting fault feature of multi-frequency signal from rotating machinery, Mechanical Systems and Signal Processing32(2012)306-319.

[28]. Z.K Peng, W.M Zhang, Z.Q Lang, G.Meng, F.L Chu, Time-frequency data fusion technique with application to vibration signal analysis, Mechanical Systems and Signal Processing29(2012)164-173.

[29]. Dongyang Dou , Jianguo Yang, Jiongtian Liu, Yingkai Zhao, A rule-based intelligent method for fault diagnosis of rotating machinery, Knowledge-Based Systems 36 (2012) 1-8.

[30]. Md. Abdul Saleem, G. Diwakar, Dr. M.R.S. Satyanarayana, Detection of Unbalance in Rotating Machines Using Shaft Deflection Measurement during Its Operation, IOSR Journal of Mechanical and Civil Engineering (IOSR-JMCE) ISSN: 2278-1684 Volume 3, Issue 3 (Sep-Oct. 2012), PP 08-20.

[31]. B. Kiran Kumar, G. Diwakar, Dr. M. R. S. Satynarayana, Determination of Unbalance in Rotating Machine Using Vibration Signature Analysis, International Journal of Modern Engineering Research (IJMER),Vol.2, Issue.5, Sep-Oct. 2012 pp-3415-3421 ISSN: 2249-6645.G.F. Bin, J.J.Gao, X.J.Li, B.S.Dhillon, Early fault diagnosis of rotating machinery based on wavelet

[32]. Zhipeng Feng, Ming Liang, Fulei Chu, Recent advances in time-frequency analysis methods for machinery fault diagnosis: review with application examples, Mechanical Systems and Signal Processing38(2013)165-205Jun Wang, Qingbo He, Fanrang Kong, Automatic fault diagnosis of rotating machines by time-scale manifold ridge analysis, Mechanical Systemsand Signal Processing 40(2013)237-256.

[33]. Qingbo He, Xiangxiang Wang, Time-frequency manifold correlation matching for periodic fault identification in rotating machines, Journal of Sound and Vibration332(2013)2611-2626.

[34]. JyotiK.Sinha, Keri Elbhbah, A future possibility of vibration based condition monitoring of rotating machines, Mechanical SystemsandSignalProcessing34(2013)231-240.

[35]. HamdiTaplak ,SelçukErkaya , Ibrahim Uzmay, Experimental analysis on fault detection for a direct coupled rotor-bearing system, Measurement 46 (2013) 336-344

[36]. R. K. Biswas , M. C. Majumdar, S. K. Basu, Vibration and Oil Analysis by Ferrography for Condition Monitoring ,J. Inst. Eng. India Ser. C (July-September 2013) 94(3):267-274

[37]. Abhisekh Bhattacharya , Pranab K. Dan, Recent trend in condition monitoring for equipment fault diagnosis, Int J SystAssurEng Management, DOI 10.1007/s13198-013-0151-z

[38]. Yaguo Lei ,Jing Lin , Zhengjia He , Ming J.Zuo, A review on empirical mode decomposition in fault diagnosis of rotating machinery, Mechanical SystemsandSignalProcessing35(2013)108-126.

[39]. Shibin Wang, GaigaiCai , Zhongkui Zhu, Weiguo Huang, Xingwu Zhang, Transient signal analysis based on LevenbergMarquardt method for fault feature extraction of rotating machines, Mechanical SystemsandSignalProcessing54-55(2015)16-40

[40]. Y. Yang, X.J.Dong, Z.K.Peng, W.M.Zhang, G.Meng, Vibration signal analysis using parameterized time-frequency method for features extraction of varying-speed rotary machinery, Journal ofSoundandVibration335(2015)350-366.

[41]. Junhong Zhang, Wenpeng Ma, Jiewei Lin, Liang Ma, XiaojieJia, Fault diagnosis approach for rotating machinery based on dynamic model and computational intelligence, Measurement 59 (2015) 73-87

[42]. Wei Li, Zhencai Zhu, Fan Jiang, Gongbo Zhou, Guoan Chen, Fault diagnosis of rotating machinery with a novel statistical feature extraction and evaluation method, Mechanical SystemsandSignalProcessing50-51(2015)414-426

[43]. IoannisChatzisavvas, FadiDohnal, Unbalance identification using the least angle regression technique, Mechanical Systems and Signal Processing 50-51(2015)706-717.

[44]. Man Shan Kan n, Andy C.C.Tan, Joseph Mathew, A review on prognostic techniques for non-stationary and non-linear rotating systems, Mechanical SystemsandSignalProcessing62-63(2015)1-20

[45]. Saurabh Singh, Dr. Manish Vishwakarma, A Review of Vibration Analysis Techniques for Rotating Machines, International Journal of Engineering Research \& Technology (IJERT), ISSN: 2278-0181, IJERTV4IS030823, Vol. 4 Issue 03, March-2015. 
[46]. HocineBendjama, Mohamad S. Boucherit, Saleh Bouhouche, Fault Diagnosis of rotating Machinery using wavelet transform and principal component analysis, Unitée de RechercheAppliquée en Sidérurgie et Métallurgie URASM-CSC, BP 196 Site Sidérurgiqued'El-hadjar, Annaba, Algérie, Département de génieélectrique et automatique, EcoleNationalePolytechnique ENP,10 HassenBadi, BP 182 El-Harrach, Alger, Algérie.

[47]. Phadatare H. P., BarunPratiher, Nonlinear Frequencies and Unbalanced Response Analysis of High Speed Rotor-Bearing Systems, Procedia Engineering 144 (2016) $801-809$.

[48]. Yanxue Wang, Jiawei Xiang, Richard Market, Ming Liang, Spectral kurtosis for fault detection, diagnosis and prognostics of rotating machines: A review with applications, Mechanical SystemsandSignalProcessing66-67(2016)679-698. 